\title{
Preemptive use of recombinant activated factor VII: many questions but few answers
}

\author{
Charles Marc Samama MD PhD FCCP
}

$\mathrm{R}$

ECOMBINANT activated factor VII (rVIIa) is a prohemostatic agent, which was developed for patients with congenital bodies toward factors VIII or IX. ${ }^{1}$ It acts locally at the site of tissue injury and vascular wall disruption, where it binds to exposed tissue factor, giving rise to small amounts of thrombin that activate platelets. It then interacts directly or indirectly with the platelet surface, amplifying coagulation and generating a thrombin burst. ${ }^{1,2}$ Recombinant activated factor VII thus plays a major role in hemostasis resulting in platelet activation, and in the final conversion of fibrinogen into fibrin. In addition rVIIa interferes with fibrinolysis when activating the thrombin-activatable fibrinolysis inhibitor and, as a result, stabilizes the fibrin clot. ${ }^{2}$

Recently, rVIIa has been proposed in a wide variety of surgical settings to decrease intraoperative bleeding and the need for transfusion. ${ }^{3}$ This has been largely prompted by the limited availability of human blood transfusion products, and their potential risks (viral, bacterial, immunologic) have lead to focus on rVIIa as a potential substitute. This new hemostatic agent has shown considerable efficacy when used compassionately in multitrauma patients, ${ }^{4}$ cardiac surgery ${ }^{5}$ and obstetrics. ${ }^{6}$ Recent case reports and small patient series have even presented the compound as a "magic bullet" ${ }^{7,8}$ However, this efficacy has not been totally borne out on prophylactic rVIIa use in scheduled surgical patients. In patients undergoing liver transplantation ${ }^{9}$ or major orthopedic surgery, ${ }^{10}$ no definite conclusion on rVIIa efficacy can yet be drawn.

In this issue of the Journal, Ballen et al. ${ }^{11}$ report the preemptive use of rVIIa in an 83-yr-old Jehovah's Witness patient who underwent emergency surgery and cardiopulmonary bypass with hypothermic arrest for aortic dissection. Despite adequate intraoperative hemostasis, rVIIa $90 \mu \mathrm{g} \cdot \mathrm{kg}^{-1}$ was administered post- cardiopulmonary bypass. No blood products were given. Recombinant activated factor VII had already been proposed in this setting but during a normothermic procedure. ${ }^{12}$ Preventing bleeding in this way in a high risk patient is a highly speculative albeit interesting procedure; it raises many questions to which there are but few answers.

First, case law which is used to judge transfusion refusal by Jehovah's Witnesses varies among countries. ${ }^{13-16}$ A physician should comply with a patient's wishes and try to avoid transfusion by making use of all available techniques. Respecting all religions is a commitment of medical ethics. However, physicians, and especially anesthesiologists working in emergency theaters, fight daily to keep their patients alive. When a patient's life is obviously jeopardized by active hemorrhage, it can be hard to accept a patient's refusal of a blood transfusion. In some European countries, courts have ruled in favour of rescue transfusions. In these countries therefore, dedicated platelet, plasma and red blood cell transfusion could have been used to control bleeding in such a patient, unless the bleeding was uncontrolled.

Second, whether any bleeding would have occurred if the patient had not received rVIIa is not certain. An alternative approach would have been to infuse rVIIa only in the event of bleeding, as described above. In the more unlikely case of no bleeding, no preemptive rVIIa administration could have been a logical decision. The efficacy of preemptive rVIIa administration was uncertain because, even though results for the compassionate use of rVIIa seem promising, those for prophylactic use of rVIIa are questionable.

Third, rVIIa might have activated the coagulation process, as reported with aprotinin, another hemostatic agent, in the same setting. Aprotinin has been considered a factor responsible for promoting disseminated intravascular coagulation (DIC) in patients

From the Department of Anesthesiology and Intensive Care, Hôpital Avicenne, Bobigny, France.

Address correspondence to: Dr. Charles Marc Samama, Department of Anesthesiology and Intensive Care, Hôpital Avicenne, 125 route de Stalingrad, 93009 Bobigny, France. Phone: +33 148955591; Fax: +33 148955589; E-mail: cmsamama@invivo.edu 
undergoing aortic repair under hypothermic cardiac arrest. ${ }^{17}$ Even if rVIIa and aprotinin have different mechanisms of action, the possibility of DIC could not be excluded. In addition, since aortic dissections generally occur in hypertensive and/or polyvascular patients, rVIIa administration could have been potentially harmful in an elderly patient, as non-DIC-related thrombotic events could not be ruled out.

The authors' decision to use rVIIa preemptively in this very special case opens an interesting debate. It cannot be established on the basis of isolated case reports whether such is more harmful than efficacious, or vice-versa. Similar case reports will no doubt be published, generating a risk that rVIIa will become a major weapon in the Jehovah Witnesses' bloodless surgery strategy. Even if numerous ongoing studies help to clarify the role of rVIIa in the near future, physicians will have to remain cautious about the compassionate use of rVIIa in a non-bleeding patient.

\section{L'usage préventif du facteur VII recombi- nant activé : beaucoup de questions, peu de réponses}

Le facteur VII recombinant activé (rVIIa) est un agent prohémostatique, élaboré pour les patients atteints d'hémophilie congénitale ou acquise, qui inhibe les anticorps anti facteur VIII ou IX. ${ }^{1}$ Il agit localement au site de la lésion tissulaire et de la rupture de la paroi vasculaire où il se lie au facteur tissulaire, générantde petites quantités de thrombine qui activent les plaquettes. Il interagit ensuite directement ou indirectement avec la surface plaquettaire, amplifiant la coagulation et générant une production de thrombine. $^{1,2}$ Le rVIIa joue ainsi un rôle important dans l'hémostase, provoquant l'activation des plaquettes, et dans la conversion finale du fibrinogène en fibrine. De plus il interfêre avec la fibrinolyse en activant l'inhibiteur de la fibrinolyse activable par la thrombine et, en conséquence, il stabilise le caillot fibrineux. ${ }^{2}$

Récemment, le rVIIa a été proposé dans maintes occasions pour réduire les saignements peropératoires et la nécessité de transfusion. ${ }^{3}$ Ce qui a été largement guidé par le manque de dérivés sanguins humains pour la transfusion dont les risques potentiels (viral, bactérien, immunologique) ont beaucoup attiré l'attention vers le rVIIa comme substitut possible. Ce nouvel agent hémostatique s'est révélé très efficace en usage humanitaire chez des polytraumatisés, en cardiochirurgie ${ }^{5}$ et en obstétrique. ${ }^{6}$ De récentes observations et de petites séries ont même présenté le composé comme une «solution magique».7,8 Mais son efficacité préventive n'a pas été totalement confirmée en chirurgie réglée. Aucune conclusion définitive ne peut être tirée sur son efficacité en cas de greffe du foie ${ }^{9}$ ou de chirurgie orthopédique majeure. ${ }^{10}$

Dans le présent numéro du Journal, Ballen et coll. ${ }^{11}$ présentent l'usage préventif du rVIIa chez une patiente de 83 ans, Témoin de Jéhovah, opérée d'urgence sous circulation extracorporelle avec asystolie sous hypothermie pour dissection aortique. Malgré l'hémostase peropératoire adéquate, $90 \mu \mathrm{gg} \cdot \mathrm{kg}^{-1} \mathrm{de}$ rVIIa ont été administrés après la circulation extracorporelle. Aucun produit sanguin n'a été transfusé. L'utilisation du rVIIa avait déjà été suggérée dans ce contexte, mais pendant une opération normothermique. ${ }^{12}$ La prévention hémorragique ainsi réalisée chez une patiente à haut risque est très hypothétique, quoique très intéressante ; elle soulève de nombreuses questions auxquelles il n'y a que peu de réponses.

Premièrement, la jurisprudence utilisée pour juger du refus des transfusions par les Témoins de Jéhovah varie d'un pays à l'autre. ${ }^{13-16}$ Un médecin peut respecter les volontés d'un patient et tenter d'éviter la transfusion en utilisant toutes les techniques disponibles. Le respect de toutes les religions est une règle du code de déontologie. Toutefois, les médecins, et surtout les anesthésiologistes qui travaillent à l'urgence, luttent chaque jour pour garder leurs patients en vie. Quand survient une hémorragie active majeure, il peut être difficile d'accepter que le patient refuse la transfusion sanguine. Dans certains pays européens, les cours imposent les transfusions de secours. Ainsi, on a pu y utiliser des transfusions spéciales de plaquettes, de plasma et de globules rouges pour contrôler les hémorragies, sauf si le saignement était incontrôlable.

Deuxièmement, il n'est pas certain qu'une hémorragie serait survenue si la patiente n'avait pas reçu de rVIIa. On aurait pu, alors perfuser du rVIIa en cas d'hémorragie seulement, comme c'est décrit plus haut. Dans le cas plus improbable d'absence d'hémorragie, une décision logique aurait été de ne pas administrer de rVIIa en prévention. L'efficacité de l'administration préventive de rVIIa était incertaine parce que, même si les résultats de l'usage humanitaire 
semble prometteur, ceux d'un usage préventif sont contestables.

Troisièmement, le rVIIa aurait pu, dans la même situation, activer le processus de coagulation, comme dans le cas d'un autre agent hémostatique, l'aprotinine. L'aprotinine a été considérée comme un facteur responsable de la coagulation intravasculaire disséminée (CIVD) chez des patients qui subissent une réparation aortique sous asystolie hypothermique. ${ }^{17}$ Même si le rVIIa et l'aprotinine ont des mécanismes d'action différents, la possibilité de CIVD ne peut être exclue. En outre, comme les dissections aortiques surviennent généralement dans des cas d'hypertension et/ou d'atteinte polyvasculaire, l'administration de rVIIa aurait pu être potentiellement dommageable chez un patient âgé, car des événements thrombotiques non reliés à la CIVD ne peuvent être exclus.

La décision des auteurs de faire un usage préventif du rVIIa dans ce cas très spécial ouvre un débat intéressant. On ne peut établir, à partir de présentations de cas isolées, si la démarche utilisée est plus dangereuse qu'efficace, ou inversement. D'autres cas similaires seront sans doute présentés, avec le risque que le rVIIa devienne une arme essentielle dans la stratégie des Témoins de Jéhovah pour une chirurgie sans transfusion. Même si de nombreuses études en cours aident à clarifier le rôle du rVIIa dans un proche avenir, les médecins devront demeurer prudents quant à l'usage humanitaire du rVIIa en l'absence d'hémorragie.

\section{References}

1 Hedner U. Recombinant activated factor VII as a universal haemostatic agent. Blood Coagul Fibrinolysis 1998; 9(Suppl 1): S147-52.

2 Levi M, Peters $M$, Buller HR. Efficacy and safety of recombinant factor VIIa for treatment of severe bleeding: a systematic review. Crit Care Med 2005; 33 : 883-90.

3 Martinowitz U, Holcomb JB, Pusateri AE, et al. Intravenous rFVIIa administered for hemorrhage control in hypothermic coagulopathic swine with grade $\mathrm{V}$ liver injuries. J Trauma 2001; 50: 721-9.

4 Dutton RP, McCunn M, Hyder M, et al. Factor VIIa for correction of traumatic coagulopathy. J Trauma 2004; 57: 709-18; discussion 718-9.

5 Karkouti K, Beattie WS, Wijeysundera DN, et al. Recombinant factor VIIa for intractable blood loss after cardiac surgery: a propensity score-matched casecontrol analysis. Transfusion 2005; 45: 26-34.

6 Bouwmeester FW, Jonkhoff AR, Verheijen RH, van Geijn $H P$. Successful treatment of life-threatening postpartum hemorrhage with recombinant activated factor VII.

Obstet Gynecol 2003; 101: 1174-6.
7 Al Douri M, Shafi T, Al Khudairi D, et al. Effect of the administration of recombinant activated factor VII (rFVIIa; NovoSeven) in the management of severe uncontrolled bleeding in patients undergoing heart valve replacement surgery. Blood Coagul Fibrinolysis 2000; 11(Suppl 1): S121-7.

8 Danilos J, Goral A, Paluszkiewicz P, Przesmycki K, Kotarski J. Successful treatment with recombinant factor VIIa for intractable bleeding at pelvic surgery. Obstet Gynecol 2003; 101: 1172-3.

9 Lodge JP, Jonas $S$, Jones RM, et al. Efficacy and safety of repeated perioperative doses of recombinant factor VIIa in liver transplantation. Liver Transpl 2005; 11 : 973-9.

10 Raobaikady R, Redman J, Ball JA, Maloney G, Grounds $R M$. Use of activated recombinant coagulation factor VII in patients undergoing reconstruction surgery for traumatic fracture of pelvis or pelvis and acetabulum: a double-blind, randomized, placebo-controlled trial. $\mathrm{Br}$ J Anaesth 2005; 94: 586-91.

11 Ballen J, Raabe M, Muirhead B. Aortic dissection, hypothermic arrest, and a Jehovah's Witness patient: a case for recombinant factor VIIa? Can J Anesth 2006; 53: 353-6.

12 Pasic M, Ruisz W, Koster A, Hetzer R. Bloodless surgery of acute type A aortic dissection in a Jehovah's Witness patient. Ann Thorac Surg 2005; 80: 1507-10.

13 Bagou $G$, Laplace MC. The anesthesiologist and a Jehovah's Witness patient (French). Ann Fr Anesth Reanim 1991; 10: 354-61.

14 Baker CE, Kelly GD, Perkins GD. Perioperative care of a Jehovah's Witness with a leaking abdominal aortic aneurysm. Br J Anaesth 1998; 81: 256-9.

15 Beauchamp TL. Methods and principles in biomedical ethics. J Med Ethics 2003; 29: 269-74.

16 Kulvatunyou N, Heard SO. Care of the injured Jehovah's Witness patient: case report and review of the literature. J Clin Anesth 2004; 16: 548-53.

17 Sundt TM 3rd, Kouchoukos NT, Saffitz JE, Murphy SF, Wareing TH, Stabl DJ. Renal dysfunction and intravascular coagulation with aprotinin and hypothermic circulatory arrest. Ann Thorac Surg 1993; 55: 1418-24. 